\title{
Image retrieval based on swarm intelligence
}

\author{
Shahbaa I. Khaleel ${ }^{1}$, Ragad W. Khaled ${ }^{2}$ \\ ${ }^{1}$ Department of Software, College of Computer Science and Mathematics, Mosul University, Iraq \\ ${ }^{2}$ Northern Technical University, Iraq
}

\begin{tabular}{l}
\hline \hline Article Info \\
\hline Article history: \\
Received Nov 6, 2020 \\
Revised May 25, 2021 \\
Accepted Jun 10, 2021 \\
\hline
\end{tabular}

Keywords:

Annular color moments

Ant colony

Image retrieval

Intelligent water drop

Swarm intelligence

\begin{abstract}
To keep pace with the development of modern technology in this information technology era, and the immense image databases, whether personal or commercial, are increasing, is requiring the management of these databases to strong and accurate systems to retrieve images with high efficiency. Because of the swarm intelligence algorithms are great importance in solving difficult problems and obtaining the best solutions. Here in this research, a proposed system is designed to retrieve color images based on swarm intelligence algorithms. Where the algorithm of the ant colony optimization (ACOM) and the intelligent water drop (IWDM) was used to improve the system's work by conducting the clustering process in these two methods on the features extracted by annular color moment method (ACM) to obtain clustered data, the amount of similarity between them and the query image, is calculated to retrieve images from the database, efficiently and in a short time. In addition, improving the work of these two methods by hybridizing them with fuzzy method, fuzzy gath geva clustering algorithm (FGCA) and obtaining two new high efficiency hybrid algorithms fuzzy ant colony optimization method (FACOM) and fuzzy intelligent water drop method (FIWDM) by retrieving images whose performance values are calculated by calculating the values of precision, recall and the f-measure. It proved its efficiency by comparing it with fuzzy method, FGCA and by methods of swarm intelligence without hybridization, and its work was excellent.
\end{abstract}

This is an open access article under the CC BY-SA license.

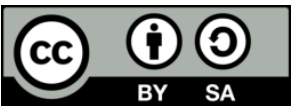

\section{Corresponding Author:}

Shahbaa I. Khaleel

Department of Software

College of Computer Science and Mathematics

Mosul University, Iraq

Email: shahbaaibrkh@uomosul.edu.iq

\section{INTRODUCTION}

Due to the rapid growth of modern technology and the uses of the World Wide Web, as well as the development of multimedia technologies, interest has increased significantly over the past few years, with digital images and the way to access images stored within a massive database as soon as possible. Where similar images are queried from the stored image database by analyzing and extracting their contents so that their retrieval will be fast and accurate [1], [2]. In the past few years, several computer vision algorithms have been developed to represent images in minimal dimensions. Machine learning algorithms have also been used in the image retrieval process, as well as in feature extraction to classify the images according to them [3]. Image retrieval systems are a serious problem due to the huge amount of information that is searched for within a very large database. Therefore, it requires efficient and high-level techniques to ensure accurate retrieval of relevant information [4], [5]. Color is one of the most important visual features that represent low-level, as it can be relied upon to differentiate between visuals by the human eye. Therefore, the 
extraction of color plays an important role in the representation of the characteristics of color images [6], [7]. In the image retrieval process, image content is dealt with, and most important features are color that enable humans to recognize images, so it is one of the common visual features used to recover color images, to ensure efficient performance of the retrieval process. There are many ways to extract features from images and they are efficient in the process of retrieving images from the database depending on the image of the query. The goal of feature extraction is to reduce the amount of data that is handled [8], [9]. Swarm based technologies have many applications within different specialties, and these technologies have been developed, and are different from one technology to another, and despite the difference, they implement the general form of these technologies. Most of these techniques have been modified based on the application used or the problem to be solved. Where the basis of work in all of them is to reach the optimal solution, that is, to reach the goal, and each technique has its own behavior to reach the solution in the search space [10]. Because of the technological development, the real world problems have become very complex, and to solve these problems with high quality, efficiency and accuracy, smart technologies have been used [11]. Clustering algorithms are used to collect data into groups, the similarity between the elements of one group is large, and the similarities between groups are few [12]. Fuzzy logic algorithms are used to perform the clustering operation and they perform much better than conventional clustering algorithms. This is because clustering algorithms have a membership function that contains membership degrees, which in turn performs a fine clustering [13].

In this research, a proposed system was designed to perform the image retrieval process based on the swarm intelligence algorithms. Where initially the features were extracted using the annular color moment method ACM method, the extraction of features is based on this method for its accuracy and obtain excellent characteristics that used in the process of retrieving color images. Where this method was applied to extract features and then its data were entries for clustering methods to ensure a retrieval of all images related to the database as per the query image provided. The clustering methods that used here are, the ant colony algorithm and intelligent water drop to perform the clustering process to reduce the time required to retrieve the images. The fuzzy clustering method fuzzy gath geva clustering algorithm (FGCA) was also used to clustered the database image features. In addition to hybridization of swarm intelligence methods with the fuzzy clustering algorithm to improve system performance. Here hybridized the FGCA with the ant colony optimization (ACOM) to produced a new method fuzzy ant colony optimization method (FACOM), and also proposed a novel method fuzzy intelligent water drop method (FIWDM).

The remainder sections of the paper are organized as follows: Section 2 provides some related work on image retrieval. Section 3 explains the method for extracting the features that used here by the research. Section 4 describes the fuzzy clustering method, section 5 present the intelligent ant colony method, and in section 6 illustrates details of the proposed method FACOM. Section 7 includes an explanation of the details of the intelligent water drop method that was used here to perform the clustering of image data, while section 8 deals the proposed hybrid method. Section 9 describes the metrics used to measure the performance of the proposed system. In section 10 the details of the proposed system are clarified. Section 11 investigates and discusses the experimental results of the proposed system, and finally in section 12 the conclusions is presented.

\section{RELATED WORKS}

As a result of the development of multimedia technologies, content-based image retrieval has become a broad field of active search for fast and accurate retrieval. Its goal is to search for and retrieve relevant images from a huge database. This topic has been taked up by many researchers, as researchers Savitha Sivan and Tusnavis Bella Mare presented an image retrieval system that relies on three methods to extract characteristics of images, namely Haralek texture descriptors, color moments, and GIST descriptions, to take advantage of many features represented in shape, color and texture. A comparison was made between these methods. In addition to, the researchers merging these methods to obtain better system performance [1]. Researcher Yingmeng Dai et al. proposed an improved algorithm for a color histogram in the HSV color system, whose subspaces are not equal quantized. This algorithm follows several steps, the first of which is the annular partition on the original image data, and then using the method presented by Aibing Rao and his group to calculate each partition. Then the distance between the distinct colored histogram is calculated. When implemented, better retrieval results are obtained [14].

A new image retrieval algorithm has been proposed by Konstantinos Konstantinidis and others, based on swarm intelligence. Whereas, the proposed method uses an ant colony algorithm in the retrieval process, which is in two stages. In the first stage, synergy of the low level descriptors, a group of ants are looking for the optimal path of the food source, where the images will represent the most similar to the query image, and here the pheromone substance is installed on every images in the high similarity region. As for the second stage of the proposed method, additional queries are made by using images that have a higher 
similarity value in the classification as new queries, and this in turn will lead to a total deposition of the pheromone substance through which performed the final retrieval [11]. Muhammad Imran and others presented a new proposed technique PSO-SVM-RF for image retrieval by combines the particle swarm optimization method with the relevance feedback RF based support vector machine SVM that reduced the interaction between the user and the system by reducing the number of RF. The proposed system was tested on large database images, and the system achieved 100\% accuracy in 8 iterations of reactions for the 10 best retrieval operations and $80 \%$ accuracy in 6 iterations for 100 retrievals. Whereas, using the proposed method, a high accuracy rate was achieved in a small number of iterations [15]. Researchers Shaimaa and Nuha presented an analytical study of the image retrieval system by adopting features extracted from images and working on cloud computing. Where the features were extracted by adopting the basic component analysis (PCA) algorithm or method because of its accurate results and the ability to deal with very large data. All the techniques and the difference between them in the speed of image retrieval are listed [16].

\section{ANNULAR COLOR MOMENT ALGORITHM ACM}

Feature extraction is the main component of all retrieval systems, as the input data included the image database and query image is converted into a set of features that are selected suitable for effective image retrieval [17]. Aim of indexing color is retrieving all images that have color combinations are similar to components of color for query image [18]. HSV color system is widely used in color vision applications. The basic components of this color system are compatible with the awareness of colors in humans. The color scheme consists of three parts: hue, saturation, value. This system of colors is used to extract the characteristics of colors, as images are converted from RGB color system to HSV color system [14]. Annular color moment according to distribution of interest points, annular division is easy to obtain. Also, we can calculate annular color histogram, that takes not only local color feature into consideration but also the space distribution information. With robustness to rotation and translation, the algorithm avoids the short-coming of losing the location information in color histogram. The annular division is easy to obtain according to the distribution of points of interest. Then, the annular color histogram is calculated, which takes the area distribution information as a complement to the local color feature, with strength of translation and rotation in addition to avoiding the loss of location information in the color histogram. The maximum radius (rad) is calculated as [8], [19], [20]:

$$
\begin{aligned}
& \text { rad }=\max _{i j \in w r}\left[(i-\text { imean })^{2}+(j-j \text { mean })^{2}\right]^{1 / 2} \\
& w l o c^{k}=\left\{i j \mid \frac{(k-1) r a d}{n} \leq\left[(i-\text { imean })^{2}+(j-j \text { mean })^{2}\right]^{1 / 2}<\frac{k \cdot r a d}{n}, i j \in w r\right\}
\end{aligned}
$$

and then calculate the following features for each interest point's $\mathrm{m}^{*} \mathrm{~m}$ neighborhood:

$$
\begin{aligned}
& f m \mu_{x y}=\frac{1}{m \cdot m} \sum_{i j \in m . m} f_{i j} \\
& s m \sigma_{x y}=\left(\frac{1}{m \cdot m} \sum_{i j \in m . m}\left(f_{i j}-f m \mu_{x y}\right)^{2}\right)^{1 / 2} \\
& t h m s_{x y}=\left(\frac{1}{m \cdot m} \sum_{i j \in m . m}\left(f_{i j}-f m \mu_{x y}\right)^{3}\right)^{1 / 3}
\end{aligned}
$$

The above features are calculated for three components of color, then calculate the annular color moment for $\mathrm{k}^{\text {th }}$ annular by using the following combination equation:

$$
\begin{aligned}
& \text { linearCM }^{k}=0.5\left(\sum_{x y \in w l o c^{k}} \text { fmuhue }_{x y}+\sum_{x y \in w l o c^{k}} \text { smohue }_{x y}+\sum_{x y \in w l o c^{k}} \text { thmshue }_{x y}\right)+ \\
& 0.25\left(\sum_{x y \in w l o c^{k}} \text { fmusat }_{x y}+\sum_{x y \in w l o c^{k}} \text { smosat }_{x y}+\sum_{x y \in w l o c^{k}} \text { thmssat }_{x y}\right)+ \\
& 0.25\left(\sum_{x y \in w l o c^{k}} \text { fmuval }_{x y}+\sum_{x y \in w l o c^{k}}\right. \text { smoval } \\
& x y \\
& \left.+\sum_{x y \in w l o c^{k}} \text { thmsval }_{x y}\right)
\end{aligned}
$$

In this research, the extraction of properties is based on this method for its accuracy and obtain excellent characteristics that used in the process of retrieving color images. Where this method was applied to extract features and then its data were entries for fuzzy cluster methods and swarm intelligence algorithms to ensure a retrieval of all images related to the database as per the query image provided. 


\section{FUZZY GATH GEVA CLUSTERING ALGORITHM FGCA}

The clustering process aims to group data into classes so that the data within a single class are similarity while they are different between the different categories. Cluster algorithms calculate the Euclidean distances between data in the solution space [12]. Fuzzy cluster has a great importance in conducting cluster data, since there is a membership function that approximates to one-class data and produces a cluster of high quality compared to traditional methods [21]. One of the important fuzzy clustering algorithms is gathgeva, where it calculates the value of cluster centers based on the following membership function [22]:

$$
m e m \mu_{i k}=\frac{1}{\sum_{j=1}^{\text {clust }}\left(\text { dist }_{i k} / \text { dist }_{j k}\right)^{2 /(m-1)}}
$$

The distance between the cluster centers and data is calculated as follows:

$$
\operatorname{dist}_{i k}\left(\text { data }_{k}, \text { center }_{i}\right)=\frac{\sqrt{\operatorname{det}\left(\text { fcov }_{m i}\right)}}{p_{i}} * \exp \left(\frac{1}{2}\left(\text { data }_{k}-\text { center }_{i}\right)^{T} f \operatorname{cov}_{m i}^{-1}\left(\text { data }_{k}-\text { center }_{i}\right)\right)
$$

where:

$$
\operatorname{fcov}_{m i}=\frac{\sum_{k=1}^{N d}\left(m e m \mu_{i k}\right)^{m}\left(\text { data }_{k}-\text { center }_{i}\right)\left(\text { data }_{k}-\text { center }_{i}\right)^{T}}{\sum_{k=1}^{N d}\left(m e m \mu_{i k}\right)^{m}}
$$

In this research, the fuzzy clustering process was used to represent each group of images through the center of their cluster instead of the original data. The query is done by comparing the characteristics of the image that extracted by ACM with the points of cluster centers of the category. The best group is then chosen according to the similarity scale used, after which the data elements belonging to these groups are retrieved.

\section{ANT COLONY OPTIMIZATION METHOD ACOM}

Ant colony optimization algorithm mimics real ant's behavior when searching for their food. Where the ants spread pheromone during search, which results in a path that affects the choice of the path of other ants. This algorithm uses digital information to represent the pheromone trails and preserves search information. The ant makes random decisions frequently until a complete solution is reached [23]. Like all swarm algorithms, this algorithm is population dependent and the benefit of that is that it allows the algorithm to use the feedback technique among members of population as a research technique [24]. The ACO algorithm relies on the ant's ability to find the shortest path from the nest to the food source. The ant moves repeatedly from one place to another to finally reach the food source. Each arc has an associated variable $\tau_{\mathrm{xy}}$ called a pheromone trail [25]. While tracing the path, the ants deposit an organic compound called pheromones. Its density is an indication of that arc's ability to build better solutions. An ant makes random decisions in each node to determine the next node. Initially a constant amount of pheromone is given to all arcs. The probability of an ant at $n_{x}$ is to select $n_{y}$ using the path pheromone value as in (10),

$$
p_{x y}^{a}(t)=\frac{\left(\tau_{x y}(t)\right)^{\alpha} \cdot\left(\gamma_{x y}(t)\right)^{\beta}}{\sum_{n_{a} \in N_{\text {allwed }}^{a}}\left(\tau_{x a}(t)\right)^{\alpha} \cdot\left(\gamma_{x a}(t)\right)^{\beta}} \quad \text { if } n_{y} \in n_{\text {allowed }}^{a}
$$

where $n_{\text {allowed }}^{a}$ represent the set of feasible grid that ant a arrives at $\mathrm{n}_{\mathrm{x}}$

In each step, the pheromone level is updated as (11),

$$
\tau_{x y}(t+1)=\rho \tau_{x y}(t)+\Delta \tau_{x y}(t)
$$

where the value of $\rho$ between 0 and $1,1-\rho$ is pheromone evaporation rate, and the value of $\Delta \tau_{x y}$ is according the performance of each ant [26]. In this research, this algorithm was used to perform the clustering process of image data, as calculated the Euclidean distance between the cluster centers for each ant which are 10 centers here because we have 10 category and image data represented by the characteristics that were extracted using the ACM algorithm.

\section{THE NEW METHOD: FUZZY ANT COLONY OPTIMIZATION METHOD FACOM}

Swarm Intelligence algorithms are powerful and efficient algorithms for resolving complex problems with high accuracy. Despite the efficiency of the ant colony algorithm used in this research, it 
needs to be developed and improved to get the best results. To develop and improve the work of this algorithm and to increase the quality and optimization of the solutions it produces, it was hybridized with fuzzy logic using the membership function of the FGCA algorithm to improve the solution. Where the membership function has been added because of its importance in helping to classify the data correctly because it contains a membership degree for each element of the images to be classified during the retrieval process. The membership function was added to the equation for adjusting the pheromone level of the ant colony algorithm as in (12).

$$
\tau_{x y}(t+1)=\rho \tau_{x y}(t) \cdot m e m \mu_{i k}+\Delta \tau_{x y}(t)
$$

This improved the image retrieval process using this hybrid method, which we named the fuzzy Ant colony optimization method. This is because the clustering process was improved by adding the membership function to the pheromone update equation, which led to the acceleration and efficiency of work.

\section{INTELLIGENT WATER DROP METHOD IWDM}

This algorithm is considered one of the algorithms of swarm intelligence, and it is one of the algorithms that depend on the population in its work [27], and it is inspired by nature as it depends on some of the basic elements of the natural water drop and its behavior by doing an action and reaction that occurs between a drop of water and the bed of the river that it is flow in it. Where the natural river often finds good paths among the many paths available in front of it on the way from the source to the destination (downstream). And the artificial water drop collaborate with each other to change their environment to reveal the best path, represented by the path that has the least soil [28]. Each intelligent drop of water has probability to choose the next node using (13)-(15) [29]:

$$
\begin{aligned}
& p_{x}(y)=\frac{f\left(\text { soilpath }_{x y}\right)}{\left.\sum_{k \in f i t v i s i t} f \text { soilpath }_{x k}\right)} \\
& f\left(\text { soil }_{x y}\right)=\frac{1}{0.01+g\left(\text { soil }_{x y}\right)} \\
& g\left(\text { soilpath }_{x y}\right)=\left\{\begin{array}{ll}
\text { soilpath }_{x y} & \text { if } \min \left(\text { soilpath }_{x k}\right) \geq 0 \\
\text { soilpath }_{x y}-\min \left(\text { soilpath }_{x k}\right) & \text { otherwise }
\end{array}\right\}
\end{aligned}
$$

The velocity of the water drop is adjusted as it moves from node $\mathrm{x}$ to node $\mathrm{y}$ as (16).

$$
\operatorname{velocity}(t+1)=\operatorname{velocity}(t)+\frac{a v}{b v+c v * \operatorname{soilpath}_{x y}^{2 \beta}}
$$

Then the soil of the water drop is adjusted that Moving from node $\mathrm{x}$ to node $\mathrm{y}$ by (17)-(19):

$$
\begin{aligned}
& \text { soil }=\text { soil }+\Delta \text { soilpat }_{x y} \\
& \Delta \text { soilpath }_{x y}=\frac{a s}{b s+c s * T_{x y}^{2}} \\
& T_{x y}=\frac{\text { dist }_{x y}}{\text { velocity }(t+1)}
\end{aligned}
$$

where: $a s, b s, c s$ was represented soil update parameter. Then adjust the soil along the path as (20).

$$
\text { soilpath }_{x y}=(1-\rho) . \text { soilpath }_{x y}-\rho \cdot \Delta \text { soilpath }_{x y}
$$

After obtaining the best solution, the soil is modified along the path by (21), which depends of the water drop soil and the soil of path.

$$
\text { soilpath }_{x y}=(1+\rho d) \text {. soilpath } x y-\rho d \cdot \frac{1}{\text { Nbest-1 }} \cdot \text { soil }
$$

where $\rho d$ represent global soil parameter, Nbest represent the nodes number of the best solution in the iteration [29]. 
The strategy for working this method is very similar to the ant colony optimization method. Where the pheromones are deposited on the ant's path of movement, and the pheromone decreases with time by evaporating. In the case of intelligent water drop method, from the path the soil is removed when the water drop moves through it. The difference is only in the path changes, as it was constant in the case of the ant colony optimization while in the case of intelligent water drop these changes depend on the speed and soil acquired by the water drop. In this research the intelligent water drop method is efficient and it is better than ant colony optimization method.

\section{NOVEL METHOD: FUZZY INTELLIGENT WATER DROP METHOD FIWDM}

Despite the efficiency of the intelligent water drop algorithm used in this research and achieving great results, it was almost better than the ant colony method, since the retrieved images are all related (relevant) even if not all the images in the database, meaning that it is less than them by a very small number and does not retrieve any unrelated (irrelevant) image and therefore I got better at working here than an ant colony method. The reason for this was the continuous modification of the soil of the water drop and the soil of the path the drop is taking. However, it needs to be developed and improved to get the best results and retrieve all the images related to the database. To develop and improve the work of this method and to increase the quality and optimization of the solutions it produces, it was hybridized with fuzzy logic using the membership function of the FGCA algorithm to improve the solution. Where the membership function has been added because of its importance in helping to classify the data correctly because it contains a membership degree for each element of the images to be classified during the retrieval process. The membership function was added to the equation for adjusting the soil of the water drop and the equation for adjusting the soil along the path as (22).

$$
\text { soil }=\text { soil. } m e m \mu_{x k}+\Delta \text { soilpath } h_{x y}
$$

Then adjust the soil along the path as (23):

$$
\text { soilpath }_{x y}=(1-\rho) . \text { soilpath }_{x y} \cdot m e m \mu_{x k}-\rho . \Delta \text { soilpath }_{x y}
$$

This enhancement the images retrieval process using this hybrid method, which we named the fuzzy intelligent water drop optimization method FIWDM. Which achieved a $100 \%$ retrieval rate.

\section{PERFORMANCE EVALUATION}

To assess the performance and effectiveness of the image retrieval process, the value of precision and recall is calculated as follows [10], [30]:

$$
\begin{aligned}
& \text { precision }=\frac{\text { number of retrieved relevant }}{\text { number of retrieved }} \\
& \text { recall }=\frac{\text { number of retrieved revelant }}{\text { number of revelant in database }}
\end{aligned}
$$

$f-$ measure it is a measure of the accuracy of test, that combines recall and precision, and it is considered a harmonic mean [1].

$$
f-\text { measure }=2 * \frac{\text { precision } * \text { recall }}{\text { precision }+ \text { recall }}
$$

\section{THE PROPOSED SYSTEM}

The proposed system in this research consists of several basic steps of work, the first of which is the process of preprocess image data to perform the retrieval process in an efficient and accurate way, using the method of extracting the characteristics of color images based on the color of the image and its details through the ACM that has proven effective here, the next step is a time-reduction procedure The retrieval process by performing the clustering process for the traits resulting from the previous step using fuzzy cluster algorithm represented by FGCA. The swarm intelligence algorithms were used to perform the clustering process ACOM as well as IWDM, and also the proposed hybridized methods FACOM, FIWDM, and proven the accuracy to work. Then, the amount of similarity between the query image and the database images is 
calculated by treating the query image characteristics with the clustering results data, i.e. the cluster centers, where the similarity is calculated between them, and the group of images belonging to the best similarity is retrieved, this is done for all the cluster methods used in the research. As this process helped to reduce the time taken to perform the recovery process for the images and also led to the accuracy of the results as only images related to the image of the query are retrieved despite the presence of many images in the database. Thus, the proposed system in this research proved its efficiency and accuracy of work. The Figure 1 illustration the steps of proposed system.

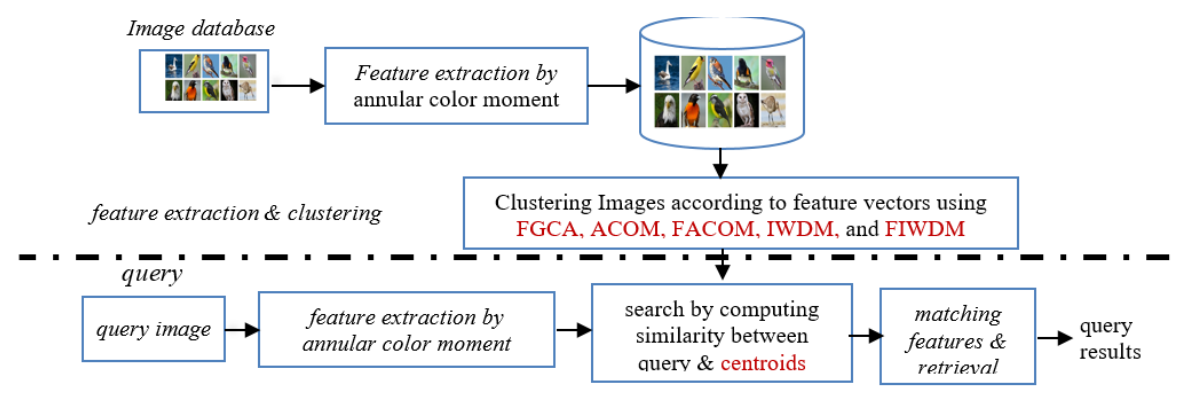

Figure 1. The steps of proposed system

\section{EXPERIMENTAL AND RESULTS}

The images database used in this research consists of ten categories, For bird types, they are, Albatross, American Goldfinch, American Kestrel, American Redstart, Annas Hummingbrid, Bald Eagle, Baltimore Orioe, Bananaquit, Barn Owl, and Bar-Tailed Godwit. As there are ten classes and each class is include a group of images as shown in Table 1 . And the size of the images used here is $224 * 224$. As shown in the Figure 2.

In this research the above color database images were used to retrieve the images by all approved, proposed and hybrid research methods. Where the ACM method was used in the process of extracting the properties of the image because of its importance in giving accurate results to all the images of the database and calculating the amount of similarity between them and the image of the query where it gave good results. But to improve the system performance, the clustering process was used on the characteristics resulting from the ACM algorithm Where it was clustered and the number of cluster centers was the number of images categories used and was equal to ten categories and after obtaining the final clusters centers the amount of similarity is calculated between the characteristics of the query image and the centers of clusters, which has the largest similarity between it and the image of the query, its images are retrieved and this was reduced the time needed to retrieve the images as well as reduce the comparison processes. Here, the FGCA fuzzy clustering method was used and gave good results compared to the first method that retrieved without the clustering method. Hence, swarm intelligence methods were used in the clustering process, where the ACOM method was used and gave better results than the fuzzy method but did not give the desired results, as it was hybridized with fuzzy logic by adding an membership function to its equations and launched the FACOM on it and in turn its results were excellent. The Table 2 shows the results of all these methods, and the precision and recall scale was used, as well as the f measure to evaluate the quality of the methods used. A query image was chosen from each image category, and the rel-ret symbol represents the number of related images from the retrieved images, and the ret symbol represents the total number of retrieved images resulting from the implementation, rec. and pre. represents the recall and precision rate respectively.

Table 1. Categories of images database

\begin{tabular}{cc}
\hline Category & Number of images \\
\hline Albatross & 30 \\
American Goldfinch & 30 \\
American Kestrel & 30 \\
American Redstart & 20 \\
Annas Hummingbrid & 30 \\
Bald Eagle & 30 \\
Baltimore Orioe & 25 \\
Bananaquit & 24 \\
Barn Owl & 30 \\
Bar-Tailed Godwit & 25 \\
\hline
\end{tabular}

Int J Elec \& Comp Eng, Vol. 11, No. 6, December 2021 : 5390 - 5401 


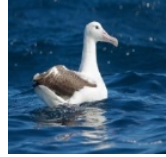

(a)

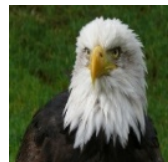

(f)

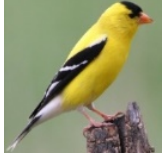

(b)

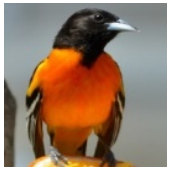

(g)

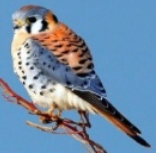

(c)

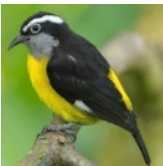

(h)

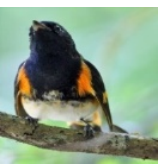

(d)

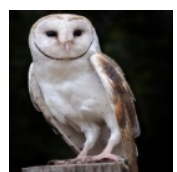

(i)

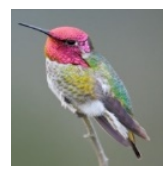

(e)

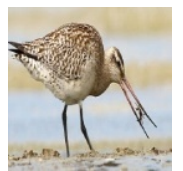

(j)

Figure 2. The images database; (a) albatross, (b) american goldfinch, (c) american kestrel, (d) american redstart,

(e) annas hummingbrid, (f) bald eagle, (g) baltimore orioe, (h) bananaquit, (i) barn owl, (j) bar-tailed godwit

Table 2. Results of ACM \& FGCA \& ACOM and FACOM methods

\begin{tabular}{lcccccc}
\hline Images & Method & rel- ret & Ret & Recall & Precision & f-measure \\
\hline ACM & 14 & 50 & 0.47 & 0.28 & 0.350933 \\
FGCA & 19 & 36 & 0.63 & 0.53 & 0.57569 \\
ACOM & 26 & 27 & 0.87 & 0.96 & 0.912787 \\
FACOM & 30 & 30 & 1.0 & 1.0 & 1.000000 \\
ACM & 16 & 43 & 0.53 & 0.37 & 0.435778 \\
FGCA & 21 & 39 & 0.7 & 0.54 & 0.609677 \\
ACOM & 27 & 27 & 0.9 & 1.0 & 0.947368 \\
FACOM & 30 & 30 & 1.0 & 1.0 & 1.000000 \\
ACM & 13 & 74 & 0.43 & 0.18 & 0.25377 \\
FGCA & 19 & 45 & 0.63 & 0.42 & 0.504 \\
ACOM & 26 & 28 & 0.87 & 0.93 & 0.899 \\
FACOM & 30 & 30 & 1.0 & 1.0 & 1.000000 \\
ACM & 6 & 35 & 0.3 & 0.17 & 0.217021 \\
FGCA & 12 & 28 & 0.6 & 0.43 & 0.500971 \\
ACOM & 17 & 17 & 0.85 & 1.0 & 0.918919 \\
FACOM & 20 & 20 & 1.0 & 1.0 & 1.000000 \\
ACM & 9 & 41 & 0.3 & 0.22 & 0.253846 \\
FGCA & 17 & 31 & 0.57 & 0.55 & 0.559821 \\
ACOM & 26 & 27 & 0.87 & 0.96 & 0.912787 \\
FACOM & 30 & 30 & 1.0 & 1.0 & 1.000000 \\
ACM & 18 & 46 & 0.6 & 0.39 & 0.472727 \\
FGCA & 21 & 43 & 0.7 & 0.49 & 0.576471 \\
ACOM & 28 & 28 & 0.93 & 1.0 & 0.963731 \\
FACOM & 30 & 30 & 1.0 & 1.0 & 1.000000 \\
ACM & 15 & 86 & 0.6 & 0.17 & 0.264935 \\
FGCA & 19 & 28 & 0.76 & 0.68 & 0.717778 \\
ACOM & 23 & 23 & 0.92 & 1.0 & 0.958333 \\
FACOM & 25 & 25 & 1.0 & 1.0 & 1.000000 \\
ACM & 8 & 57 & 0.33 & 0.14 & 0.196596 \\
FGCA & 15 & 33 & 0.63 & 0.45 & 0.525 \\
FCOM & 21 & 21 & 0.88 & 1.0 & 0.93617 \\
FCOM & 24 & 24 & 1.0 & 1.0 & 1.000000 \\
ACM & 11 & 65 & 0.37 & 0.17 & 0.232963 \\
FGCA & 18 & 42 & 0.6 & 0.43 & 0.500971 \\
FCOM & 26 & 28 & 0.87 & 0.93 & 0.899 \\
FCOM & 30 & 30 & 1.0 & 1.0 & 1.000000 \\
& 13 & 49 & 0.52 & 0.27 & 0.355443 \\
& 17 & 32 & 0.68 & 0.53 & 0.595702 \\
& 21 & 23 & 0.84 & 0.91 & 0.8736 \\
& 25 & 25 & 1.0 & 1.0 & 1.0000000 \\
\hline
\end{tabular}

Also, the swarm intelligence method IWDM, which is similar in its work to the ant colony algorithm, was used in the clustering process where its results according to the calculated measures were better than the ant colony, as the number of retrieved images are all related to the stored database images, which indicates its accuracy by performing the clustering process, despite its strength. However, it did not retrieve all database images similar to the selected query image, so it was improved by hybridizing them with fuzzy logic and obtaining a new method called FIWDM, and this method gave excellent results. The Table 3 shows the results of these methods for query images for all database image categories used by the research. 
The Table 4 shows the comparison between all the methods used in the research, as it shows the number of related images from the retrieved and the total number of retrieved images for each method, where the number 16 represents the number of related images from the retrieved in ACM method, 18 in the FGCA method, 25 in the ACOM method and 27 in the IWDM method. As for the number of images, the total retrieval is in the ACM method the number was 73 and in the FGCA equal to 33 while in the ACOM method it was equal to 26 and for the IWDM method the number of related images was 27 which equals the number of all retrieved images. As for the FACOM and FIWDM methods, the number of related images is equal to the number of total retrieved images, which in turn is equal to the number of related images in the database. Therefore, these two methods are the best ways to retrieve 100 percent of database images according to the query image. And Table 5 shows the performance of all methods that used in research, also the proposed methods FACOM and FIWDM are the best methods, where the value of precision and recall are $100 \%$. Figure 3 shows the results of all methods when applied on query image of category American Goldfinch.

Table 3. Results of ACM \& FGCA \& IWDM and FIWDM methods

\begin{tabular}{|c|c|c|c|c|c|c|}
\hline Images & Method & rel- ret & Ret & Recall & Precision & f-measure \\
\hline & $\mathrm{ACM}$ & 12 & 63 & 0.4 & 0.19 & 0.257627 \\
\hline & FGCA & 20 & 32 & 0.67 & 0.63 & 0.649385 \\
\hline & IWDM & 27 & 27 & 0.9 & 1.0 & 0.947368 \\
\hline & FIWDM & 30 & 30 & 1.0 & 1.0 & 1.000000 \\
\hline & $\mathrm{ACM}$ & 14 & 39 & 0.47 & 0.36 & 0.407711 \\
\hline & FGCA & 22 & 37 & 0.73 & 0.59 & 0.652576 \\
\hline & IWDM & 28 & 28 & 0.93 & 1.0 & 0.963731 \\
\hline & FIWDM & 30 & 30 & 1.0 & 1.0 & 1.000000 \\
\hline & $\mathrm{ACM}$ & 12 & 46 & 0.4 & 0.26 & 0.315152 \\
\hline & FGCA & 18 & 34 & 0.6 & 0.53 & 0.562832 \\
\hline & IWDM & 27 & 27 & 0.9 & 1.0 & 0.947368 \\
\hline & FIWDM & 30 & 30 & 1.0 & 1.0 & 1.000000 \\
\hline & $\mathrm{ACM}$ & 8 & 31 & 0.4 & 0.26 & 0.315152 \\
\hline & FGCA & 14 & 24 & 0.7 & 0.58 & 0.634375 \\
\hline & IWDM & 18 & 18 & 0.9 & 1.0 & 0.947368 \\
\hline & FIWDM & 20 & 20 & 1.0 & 1.0 & 1.000000 \\
\hline & $\mathrm{ACM}$ & 10 & 42 & 0.33 & 0.24 & 0.277895 \\
\hline & FGCA & 16 & 29 & 0.53 & 0.55 & 0.539815 \\
\hline & IWDM & 27 & 27 & 0.9 & 1.0 & 0.947368 \\
\hline & FIWDM & 30 & 30 & 1.0 & 1.0 & 1.000000 \\
\hline & ACM & 17 & 43 & 0.57 & 0.40 & 0.470103 \\
\hline & FGCA & 21 & 40 & 0.7 & 0.52 & 0.596721 \\
\hline & IWDM & 29 & 29 & 0.97 & 1.0 & 0.984772 \\
\hline & FIWDM & 30 & 30 & 1.0 & 1.0 & 1.000000 \\
\hline & ACM & 14 & 73 & 0.56 & 0.19 & 0.283733 \\
\hline & FGCA & 18 & 27 & 0.72 & 0.67 & 0.694101 \\
\hline & IWDM & 24 & 24 & 0.96 & 1.0 & 0.979592 \\
\hline & FIWDM & 25 & 25 & 1.0 & 1.0 & 1.000000 \\
\hline & $\mathrm{ACM}$ & 9 & 55 & 0.38 & 0.16 & 0.225185 \\
\hline & FGCA & 15 & 31 & 0.63 & 0.48 & 0.544865 \\
\hline & IWDM & 22 & 22 & 0.92 & 1.0 & 0.958333 \\
\hline & FIWDM & 24 & 24 & 1.0 & 1.0 & 1.000000 \\
\hline & $\mathrm{ACM}$ & 12 & 63 & 0.4 & 0.19 & 0.257627 \\
\hline & FGCA & 19 & 39 & 0.63 & 0.49 & 0.55125 \\
\hline & IWDM & 27 & 27 & 0.9 & 1.0 & 0.947368 \\
\hline & FIWDM & 30 & 30 & 1.0 & 1.0 & 1.000000 \\
\hline & ACM & 12 & 34 & 0.48 & 0.35 & 0.404819 \\
\hline & FGCA & 18 & 29 & 0.72 & 0.62 & 0.666269 \\
\hline & IWDM & 23 & 23 & 0.92 & 1.0 & 0.958333 \\
\hline 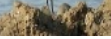 & FIWDM & 25 & 25 & 1.0 & 1.0 & 1.000000 \\
\hline
\end{tabular}

Table 4. Comparison between methods

\begin{tabular}{ccccccccc}
\hline Category & \multicolumn{2}{c}{ ACM } & \multicolumn{3}{c}{ FGCM } & \multicolumn{2}{c}{ ACOM } & \multicolumn{2}{c}{ IWDM } \\
& Rel-ret & Ret & Rel-ret & Ret & Rel-ret & Ret & Rel-ret & Ret \\
\hline Albatross & 16 & 73 & 18 & 33 & 25 & 26 & 27 & 27 \\
American Goldfinch & 15 & 46 & 15 & 38 & 23 & 25 & 26 & 26 \\
American Kestrel & 11 & 53 & 17 & 35 & 24 & 27 & 28 & 28 \\
American Redstart & 7 & 37 & 13 & 24 & 17 & 18 & 18 & 18 \\
Annas Hummingbrid & 11 & 41 & 17 & 28 & 25 & 27 & 28 & 28 \\
Bald Eagle & 17 & 49 & 22 & 41 & 27 & 28 & 29 & 29 \\
Baltimore Orioe & 16 & 77 & 18 & 27 & 23 & 23 & 24 & 24 \\
Bananaquit & 10 & 59 & 16 & 31 & 22 & 22 & 23 & 23 \\
Barn Owl & 13 & 62 & 18 & 38 & 26 & 28 & 27 & 27 \\
Bar-Tailed Godwit & 12 & 35 & 17 & 29 & 21 & 21 & 24 & 24 \\
\hline
\end{tabular}


Table 5. Performance measures for all methods

\begin{tabular}{ccccccccc}
\hline Category & \multicolumn{2}{c}{ ACM } & \multicolumn{2}{c}{ FGCA } & \multicolumn{2}{c}{ ACOM } & \multicolumn{2}{c}{ IWDM } \\
& $\mathrm{P} \%$ & $\mathrm{R} \%$ & $\mathrm{P} \%$ & $\mathrm{R} \%$ & $\mathrm{P} \%$ & $\mathrm{R} \%$ & $\mathrm{P} \%$ & $\mathrm{R} \%$ \\
\hline Albatross & 21.91781 & 53.33333 & 54.54545 & 60.0 & 96.15385 & 83.33333 & 100.0 & 90.0 \\
American Goldfinch & 32.6087 & 50.0 & 39.47368 & 50.0 & 92.0 & 76.66667 & 100.0 & 86.66667 \\
American Kestrel & 20.75472 & 36.66667 & 48.57143 & 56.66667 & 88.88889 & 80.0 & 100.0 & 93.33333 \\
American Redstart & 18.91892 & 35.0 & 54.16667 & 65.0 & 94.44444 & 85.0 & 100.0 & 90.0 \\
Annas Hummingbrid & 26.82927 & 36.66667 & 60.71429 & 56.66667 & 92.59259 & 83.33333 & 100.0 & 93.33333 \\
Bald Eagle & 34.69388 & 56.66667 & 53.65854 & 73.33333 & 96.42857 & 90.0 & 100.0 & 96.66667 \\
Baltimore Orioe & 20.77922 & 64.0 & 66.66667 & 72.0 & 100.0 & 92.0 & 100.0 & 96.0 \\
Bananaquit & 16.94915 & 41.66667 & 51.6129 & 66.66667 & 100.0 & 91.66667 & 100.0 & 95.83333 \\
Barn Owl & 20.96774 & 43.33333 & 47.36842 & 60.0 & 92.85714 & 86.66667 & 100.0 & 90.0 \\
Bar-Tailed Godwit & 34.28571 & 48.0 & 58.62069 & 68.0 & 100.0 & 84.0 & 100.0 & 96.0 \\
\hline
\end{tabular}

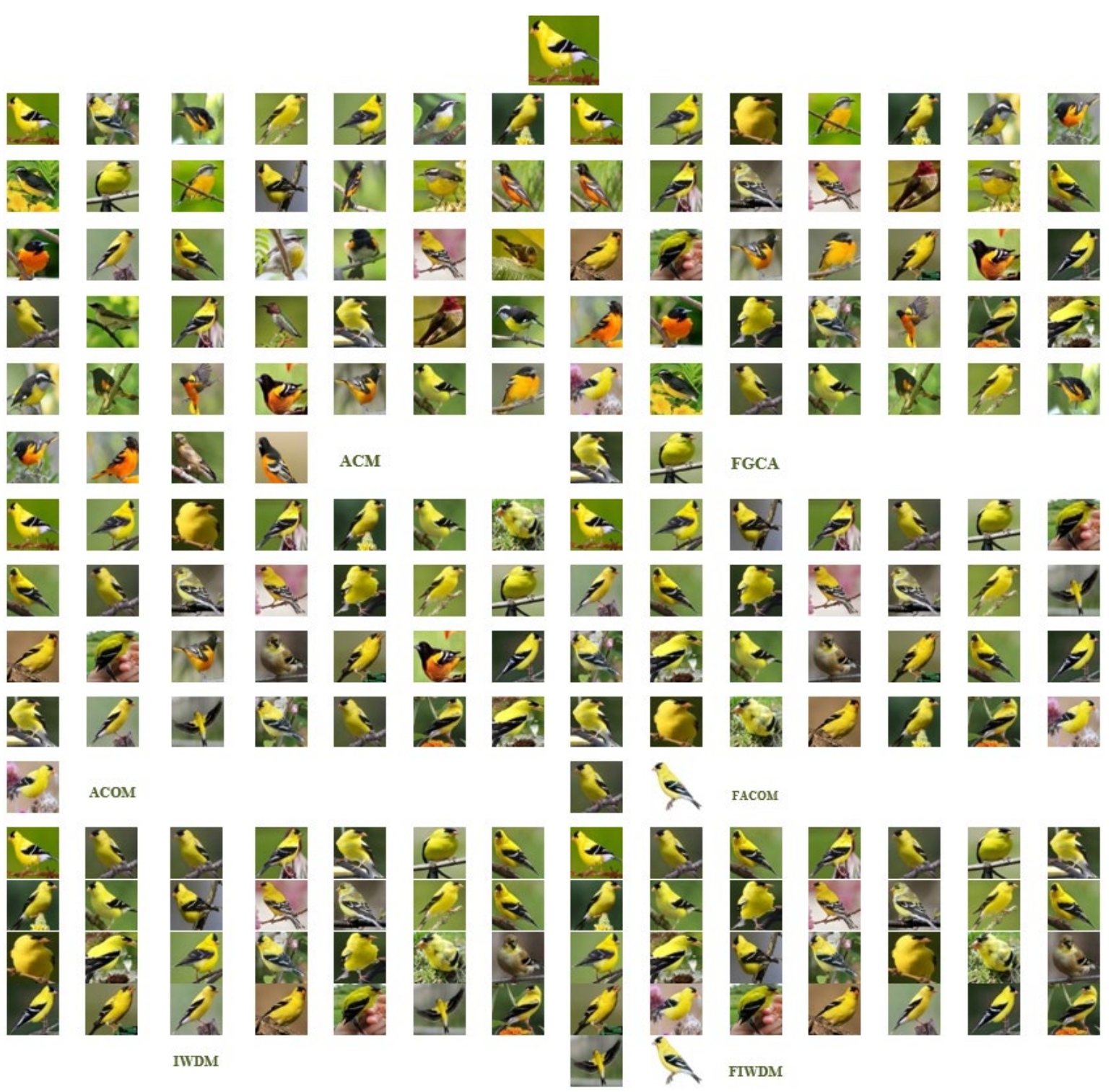

Figure 3. The results of all methods when applied on query image of category American Goldfinch

\section{CONCLUSION}

In this research, a suggested system for retrieving color images using swarm intelligence methods was built, and to improve their performance are hybridized. Where the ACM method was used in the process of extracting the features of the image because of its importance in giving accurate results to all the images of the database and to improve the performance of the system the clustering process was used on the features 
resulting from the ACM algorithm where it was clustered and the number of clusters centers was the number of image categories used, and after obtaining final clusters centers, images are retrieved based on the query image provided by the user, thus reducing the time required to retrieve the images, as well as reducing comparison operations. Here, the FGCA cluster method was used and gave good results. Hence, swarm intelligence methods were used in the clustering process, where the ACOM method was used and gave better results than the fuzzy method but did not give the desired results, as it was hybridized with fuzzy logic and called FACOM and in turn its results were excellent. Also, the IWDM swarm Intelligence method, which is similar in its work to the ant colony algorithm, was used in the clustering process where its results according to the calculated measures were better than the ant colony, as the number of retrieved images are all related to the stored database images, which indicates its accuracy by performing the clustering process, despite its strength. However, it did not retrieve all database images similar to the selected query image, so it was improved by hybridizing them with fuzzy logic and obtaining a new method called FIWDM, and this method gave excellent results. Where the Performance of system that proposed here was evaluated by calculating values of recall and precision as well as the $f$-measure.

\section{ACKNOWLEDGEMENTS}

The Author would like to Thank the Mosul University in Iraq for all supporting.

\section{REFERENCES}

[1] S. Sivan and Th. B. Mary, "An Optimized Feature Extraction Technique For Content Based Image Retrieval," International Journal of Image Processing and Vision Sciences (IJIPVS), vol. 2, no. 1, pp. 35-40, 2013, doi: 10.47893/IJIPVS.2013.1061.

[2] C. Umamaheswari, R. Bhavani and K. Thirunadana, "Texture and Color Feature Extraction from Ceramic Tiles for Various Flaws Detection Classification," International Journal on Future Revolution in Computer Science and Communication Engineering, vol. 4, no. 1, pp. 169-179, 2018.

[3] K. S. Aiswarya, N. Santhi and K. Ramar, "Content-Based Image Retrieval For Mobile Devices Using Multi-Stage Autoencoders," Journal of Critical Reviews, vol. 7, no. 6, pp. 63-69, 2020, doi: 10.31838/jcr.07.06.14.

[4] R. Inbaraj and G. Ravi, "A Survey On Recent Trends In Content Based Image Retrieval System," Journal of Critical Reviews, vol. 7, no. 11, pp. 961-965, 2020, doi: 10.31838/jcr.07.11.171.

[5] A. S. Prabuwono, W. Usino, A. Bramantoro, K. H. S. Allehaibi, A. Hasniaty and T. Defisa, "Content Based Image Retrieval and Support Vector Machine Methods for Face Recognition,” TEM Journal, vol. 8, no. 2, pp. 389-395, 2019, doi: 10.18421/TEM82-10.

[6] A. Latif et al., "Content-Based Image Retrieval and Feature Extraction: A Comprehensive Review," Mathematical Problems in Engineering, vol. 2019, pp 1-21, 2019, Art. no. 9658350, doi: 10.1155/2019/9658350.

[7] R. Rajkumar and M. Sudhamani, "Content based Image Retrieval System using Combination of Color and Shape Features, and Siamese Neural Net," International Journal of Innovative Technology and Exploring Engineering, vol. 9, no. 2S, pp. 2278-3075, 2019, doi: 10.35940/ijitee.B1053.1292S19.

[8] Z. Zhao, Q. Tian, H. Sun, X. Jin and J. Guo, "Content Based Image Retrieval Scheme using Color, Texture and Shape Features," International Journal of Signal Processing, Image Processing and Pattern Recognition, vol. 9, no. 1, pp. 203-212, 2016, doi: 10.14257/ijsip.2016.9.1.19.

[9] S. M. Singh and K. Hemachandran, "Content-Based Image Retrieval using Color Moment and Gabor Texture Feature," IJCSI International Journal of Computer Science Issues, vol. 9, no. 5, pp. 299-309, 2012.

[10] B. I. Khaleel, "Using Artificial Intelligence Techniques for Image Compression," AL-Rafidain Journal of Computer Sciences and Mathematics, vol. 11, no. 2, pp. 65-81, 2014, doi: 10.33899/csmj.2014.163750

[11] K. Konstantinidis, G. Ch Sirakoulis and I. Andreadis, "An Intelligent Image Retrieval System Based on the Synergy of Color and Artificial Ant Colonies," Scandinavian Conference on Image Analysis, vol. 4522, 2007, pp. 868-877, doi: 10.1007/978-3-540-73040-8_88.

[12] Sh. I. Khaleel and K. M. M. Saleh, "Detection of network anomaly based on hybrid intelligence techniques," $A L-$ Rafidain Journal of Computer Sciences and Mathematics, vol. 9, no. 2, pp. 81-98, 2012, doi: 10.33899/csmj.2012.163720.

[13] S. Annas, S. Nyompa, R. Arisandi, M. Nusrang and S. Eka, "Fuzzy c-means and gath-geva methods in clustering districts based on human development index (hdi) in south Sulawesi," Journal of Physics: Conference Series, vol. 1317, 2019, pp. 1-7, 2014, Art. no. 012014.

[14] Y. Dai and P. Yarlagadda, "Image Retrieval Method Based on Vision Feature of Color," Applied Mechanics and Materials, vol. 303-306, pp. 1406-1411, 2013, doi: 10.4028/www.scientific.net/AMM.303-306.1406.

[15] M. Imran, R. Hashim, A. K. N. Elaiza and A. Irtaza, "Stochastic Optimized Relevance Feedback Particle Swarm Optimization for Content Based Image Retrieval," The Scientific World Journal, vol. 2014, pp. 1-12, 2014, Art. no. 752090, doi: 10.1155/2014/752090.

[16] Sh. Shaker and N. Khassaf, "Methods of Image Retrieval Based Cloud," International Journal of Innovative Technology and Exploring Engineering, vol. 9, no. 3, pp. 636-644, 2020, doi: 10.35940/ijitee.C8139.019320. 
[17] A. Malakar and J. Mukherjee, "Image Clustering using Color Moments, Histogram, Edge and K-means Clustering," International Journal of Science and Research (IJSR), vol. 2, no. 1, pp. 532-537, 2013.

[18] D. Maity and J. Mukherjee, "A Novel Approach to Content Based Image Retrieval Using Support Vector Machine," International Journal of Advanced Information Science and Technology (IJAIST), vol. 3, no. 5, pp. 54-62, 2014.

[19] X. Zhang, B. Guo and J. Pan, "Retrieval method based on IPDSH and R/KPSO," International Journal of Innovative computing, Information and control, vol. 10, no. 3, pp. 919-931, 2014.

[20] Y. Deng and Y. Yu, "Self-feedback image retrieval algorithm based on annular color moments," EURASIP Journal on Image and Video Processing, vol. 7, pp. 1-13, 2019, doi: 10.1186/s13640-018-0400-9.

[21] B. I. AL-Taey, "Artificial Intelligent Techniques for Intrusion Detection and Classification." Ph.D. Thesis, Computer Science, College of Computer Science and Mathematics, University of Mosul, 2012.

[22] B. I. Khaleel, "Image Clustering based on Artificial Intelligence Techniques," AL-Rafidain Journal of Computer Sciences and Mathematics, vol. 11 , no. 1, pp. 99-112, 2014, doi: 10.33899/csmj.2014.163735.

[23] R. Hemalatha, G. Karthikeyan and R. Mahalakshmi, "Implementation of Aco Algorithm in Rwa," International Journal of Innovative Technology and Exploring Engineering (IJITEE), vol. 8, no. 2S2, pp. 197-200, 2018.

[24] S. I. Khaleel and R. W. Khaled, "Selection and Prioritization of Test Cases by using Bees Colony," AL-Rafidain Journal of Computer Sciences and Mathematics, vol. 11, no. 1, pp. 179-201, 2014, doi: 10.33899/csmj.2014.163746.

[25] Ch. Blum, "Ant colony optimization: Introduction and recent trends," Physics of Life Reviews, vol. 2, pp. 353-373, 2005, doi: 10.1016/j.plrev.2005.10.001.

[26] P. Li and H. Zhu, "Parameter Selection for Ant Colony Algorithm Based on Bacterial Foraging Algorithm," Mathematical Problems in Engineering, vol. 2016, pp. 1-12, 2016, Art. no. 6469721, doi: 10.1155/2016/6469721.

[27] Sh. I. Khaleel and A. A. Al Thanoon, "Design a Tool for Generating Test Cases using Swarm Intelligence," $A L-$ Rafidain Journal of Computer Sciences and Mathematics, vol. 1, no. 10, pp. 421-444, 2013, doi: $10.33899 / \mathrm{csmj} .2013 .163468$.

[28] A. Biswas and B. Biswas, "Swarm Intelligence Techniques and Their Adaptive Nature with Applications," Intelligent Soft Computations, Studies in Fuzziness and Soft Computing, vol. 319, pp. 253-273, 2015, doi: 10.1007/978-3-319-12883-2_9.

[29] G. Chen, X. Wu, J. Li and H. Guo, "Green Vehicle Routing and Scheduling Optimization of Ship Steel Distribution Center Based on Improved Intelligent Water Drop Algorithms," Mathematical Problems in Engineering, vol. 2020, pp. 1-13, 2020, Art. no. 9839634, doi: 10.1155/2020/9839634.

[30] K. Chu and G. Liu, "Image Retrieval Based on a Multi-Integration Features Model," Mathematical Problems in Engineering, vol. 2020, pp. 1-10, 2020, Art. no. 1461459, doi: 10.1155/2020/1461459. 